\title{
Reduction of plasma 1,5-anhydroglucitol (1-deoxyglucose) concentration in diabetic patients
}

\author{
T. Yamanouchi ${ }^{1}$, H. Akanuma ${ }^{2}$, T. Nakamura ${ }^{3}$, I. Akaoka ${ }^{1}$ and Y.Akanuma ${ }^{4}$ \\ ${ }^{1}$ The Second Department of Internal Medicine, University of Teikyo, \\ ${ }^{2}$ Department of Chemistry, College of Arts and Sciences, University of Tokyo, \\ ${ }^{3}$ Research Laboratories Pharmaceuticals Group, Nippon Kayaku Co., Ltd., and \\ ${ }^{4}$ Institute for Diabetes Care and Research, Asahi Life Foundation, Tokyo, Japan
}

\begin{abstract}
Summary. The plasma concentration of 1,5-anhydro-D-glucitol(AG)(1-deoxyglucose) is known to decrease in diabetic patients. In order to evaluate the usefulness of this polyol as a diabetic marker, we examined the specificity of the plasma AG reduction in various diseases: the plasma AG level was determined in 108 newly diagnosed diabetic patients, 229 normal subjects and 200 patients with various other disorders. The mean plasma AG concentration in diabetes mellitus was $1.9 \pm 1.8 \mu \mathrm{g} / \mathrm{ml}$ (mean $\pm \mathrm{SD}$ ), which was definitely lower than that in healthy subjects and patients with other diseases including some metabolic and hormonal diseases (mean value range: $13.4-28.3 \mu \mathrm{g} / \mathrm{ml}$ ). Only the "malignancies" group showed statistically different mean values from that in normal subjects; however, these values were much
\end{abstract}

higher than those of diabetic patients. The AG concentration seemed to be relatively low in some severe by uraemic patients, but is likely to be little influenced by the glomerular filtration rate. Upon adjustment for sex and age, AG concentration was not found to be correlated with the degree of obesity in both healthy subjects and diabetic patients. The plasma AG concentration showed a tendency to be higher in healthy males than in healthy females in all age-matched groups; however, statistically significant differences were not seen. Also, no significant influence of age was observed.

Key words: 1,5-anhydro-D-glucitol, 1-deoxyglucose, polyol, diabetic marker, obesity, uraemia, age.
1,5-Anhydro-D-glucitol(AG)(1-deoxyglucose, 1,5-anhydro-D-sorbitol) is a six-carbon monosaccharide resembling glucose in structure, and one of the main polyols in human cerebrospinal fluid [1] and serum [2]. The plasma AG concentration has been shown to decrease in streptozotocin-induced diabetic rats [3] and diabetic humans $[2,4-7]$. Such a low AG concentration in plasma was reversed at least in Type 2 (non-insulindependent) diabetic patients by means of strict glycaemic control [8]. Although it has been confirmed that the AG reduction is sensitive to metabolic disarrangement in diabetes mellitus $[7,8]$ no detailed study on the specificity of the low plasma AG concentration observed in diabetes mellitus has been reported. Servo et al. first reported reductions in AG concentration in cerebrospinal fluid (CSF) and plasma in several neurological diseases. They also mentioned that the plasma AG concentration was decreased in renal failure [9], whereas the serum AG level was within normal range in the sera of patients with arterial hypertension or rheumatic disease [10]. Some other reports showed that the plasma AG level was low in renal failure subjects $[9,11]$. However, these data were for a relatively small number of subjects; and only a few number of diseases were checked. Furthermore, no information has appeared on the changes in plasma AG concentration in various metabolism-altered or -related diseases, such as cirrhosis, hormonal diseases, hyperlipidaemia or gout. In this work, therefore, we extensively studied the plasma AG concentration in patients with various diseases, and also examined the correlation of the AG concentration with the degree of obesity, age and sex.

\section{Subjects and methods}

\section{Subjects and diagnosis}

The plasma AG concentration levels were examined in 108 newly diagnosed patients ( 58 males and 50 nonpregnant females), 18 to 80 (mean age, 48 years), who were referred for glucose tolerance tests (GTT). The normal control subjects consisted of 229 subjects (123 males and 106 nonpregnant females; mean age, 47 years) with no history or clinical evidence of diabetes mellitus, liver disease, renal disease, anaemia or gastrointestinal disease. They were ascertained to be free from these disorders on the basis of laboratory data. They were not taking any medication that might have affected glucose metabolism. Classification as diabetic or non-diabetic subjects 
Table 1. Plasma 1,5-anhydroglucitol (AG) levels in healthy subjects and patients with various disorders

\begin{tabular}{lcll}
\hline Diagnosis & $\begin{array}{l}\text { No. of cases } \\
\text { (male/female) }\end{array}$ & $\begin{array}{l}\text { Mean age } \\
\text { (years) }\end{array}$ & $\begin{array}{l}\text { Plasma AG } \\
(\mu \mathrm{g} / \mathrm{ml}) \\
(\mathrm{mean} \pm \mathrm{SD})\end{array}$ \\
\hline $\begin{array}{l}\text { Healthy subjects } \\
\text { Cirrhosis }\end{array}$ & $229(123 / 106)$ & 47 & $23.8 \pm 7.2$ \\
Renal failure & $20(12 / 8)$ & 50 & $21.9 \pm 7.2$ \\
Benign gastrointesti- & $13(16 / 13)$ & 51 & $20.5 \pm 6.8$ \\
$\quad$ nal diseases & & 46 & $24.2 \pm 5.6$ \\
Collagen diseases & $4(2 / 2)$ & 40 & $17.8 \pm 6.6$ \\
Leukaemia & $9(5 / 4)$ & 47 & $17.7 \pm 5.7$ \\
Malignancies & $17(10 / 7)$ & 54 & $13.4 \pm 8.1^{\mathrm{b}}$ \\
Miscellaneous & $21(11 / 10)$ & 48 & $22.1 \pm 5.9$ \\
Insulinoma & $4(2 / 2)$ & 47 & $16.7 \pm 6.1$ \\
Gout & $36(33 / 3)$ & 53 & $28.3 \pm 9.2$ \\
Hypercholesteraemia & $29(17 / 12)$ & 49 & $22.7 \pm 6.4$ \\
Hyper- or hypo- & $18(9 / 9)$ & 45 & $22.1 \pm 6.8$ \\
$\quad$ thyroidism & $108(58 / 50)$ & 48 & $1.9 \pm 1.8^{\mathrm{a}}$ \\
Diabetes & & & \\
\hline
\end{tabular}

Scheffe's test for single subgroups: ${ }^{a} p<0.001 ;{ }^{b} p<0.01$ (vs healthy subjects)

was based on the results of a standard 2-h oral 75-g glucose loading according to the criteria established by the National Diabetes Data Group [12] and the World Health Organisation Expert Committee [13]. The non-diabetic group mainly comprised people who came to the hospital for thorough physical examinations. Plasma was also collected from patients with various disorders, which are listed in Table 1. In this table, the "Miscellaneous" group mainly comprised cases of respiratory diseases, gallstones, neurological diseases and hypertension. The "Malignancies" group mainly comprised cases of lung carcinomas, hepatomas, gastric carcinomas and breast cancer. All these groups were not statistically different as to mean age or gender distribution except for the gout group in which males were predominant: No member in these groups had a personal or familial history of diabetes, and randomly checked blood glucose concentrations were normal. Every patient showed the normal 75-g GTT pattern, except the leukaemia and malignancy group patients, with whom we did not perform glucose load. The correlation of the AG concentration with the degree of obesity was examined in 122 subjects selected from the normal control subjects, aged from 30 to 60 years. These subjects were classified into five groups: highly obese ( 4 males, 3 females), moderately obese ( 12 males, 11 females), standard ( 27 males, 24 females), moderately lean (16 males, 14 females) and highly lean ( 5 males, 6 females). The degree of obesity was expressed as the body mass index $\left(\mathrm{BMI}, \mathrm{kg} / \mathrm{m}^{2}\right)$. Comparison of the $A G$ concentration with the degree of obesity was also performed among 111 Type 2 patients under glycaemic control to various extents. These Type 2 diabetic patients ( 5 were being treated with insulin plus sulfonylureas, 51 with a diet and sulfonylureas, and 55 with diet only) were selected from among patients who had shown stable glycaemic control for at least the previous four months, that is, the variance of $\mathrm{HbA}_{1}$ levels was within $\pm 10 \%$ during that period. Type 2 diabetic patients had neither ketoacidosis within the first year after diagnosis, nor a permanent need for insulin. Their diabetic subclass was also confirmed by the results of analysis of the free C-peptide response to glucagon, as described previously [14].

\section{Assays}

The glucose concentration was determined with a FUII DRI-CHEM 2000 analyser (Fuji Photo Film Co., Tokyo, Japan) [15]. Glycosylated haemoglobin was assayed using a column system provided by BioRad Laboratories (Richmond, Calif, USA). All column assays were carried out in a water bath maintained at $23^{\circ} \mathrm{C}$, and equality control was assessed on the basis of the variability of data for fresh normal samples. The standard glucagon test was performed according to Poulsen et al. [16], and serum free C-peptide reactivity (CPR) was determined according to the method described by Hsieh and Akanuma [14].

The plasma AG concentration was determined by gas liquid chromatography (GLC) as described previously [8]. In brief, an aliquot of a venous plasma specimen $(0.1 \mathrm{ml})$ was added to an internal standard, xylitol, and then deproteinised with methanol. The methanolic extract thus obtained was dried and the hydrophobic material was removed by partition between water and chloroform. The aqueous layer was dried and the resulting residue acetylated. The acetylated material was extracted with ether and then subjected to isothermic GLC $\left(203^{\circ} \mathrm{C}\right)$ on SP $2340 /$ Chromosorb W packed in a 2-m glass column. The gas chromatograph used was a Model GC4CMPF (Shimadzu Seisakusho, Ltd., Kyoto, Japan). The coefficient of variation (CV) calculated from the data for the standard was $3.9 \%$. The CV within a single assay set was $1.4 \%$.

\section{Statistical analysis}

All data were stored on an NEC PC-9801 computer for analysis.

Differences between groups were estimated by Scheffe's test for single subgroups. Differences were defined as significant when $p<$ 0.05 .

\section{Results}

\section{Plasma AG concentration in diabetes mellitus and various diseases}

The data given in Table 1 show that the distribution of the plasma AG concentration in the newly diagnosed diabetic patients was significantly different from that in the normal control subjects and the cases of other diseases. The mean plasma AG concentration in diabetes mellitus was $1.9 \pm 1.8 \mu \mathrm{g} / \mathrm{ml}$ (mean $\pm \mathrm{SD}$ ), which was definitely lower than that in other various diseases (mean value range for these diseases: $13.4-28.3 \mu \mathrm{g} / \mathrm{ml}$ ) and normal healthy subjects $(23.8 \pm 7.2 \mu \mathrm{g} / \mathrm{ml})$. As to the mean plasma AG concentration, the five groups malignancies, leukaemia, collagen diseases, insulinoma and gout patients - were different from the healthy subjects. However the statistical difference was observed only in the "malignancies" group $(p<0.01)$ (Table 1). The distributions of the AG concentration in various diseases were also very wide as in the healthy subjects. Assuming $7.5 \mu \mathrm{g}$ (mean $+3 \mathrm{SD}$ ) to be the diagnostic limit for diabetes mellitus, all 108 diabetic patients could be distinguished from normal patients, as described in our previous report; and nearly all the cases with the various other diseases could also be separated from the diabetes mellitus cases except $18 \%$ of those in the malignancy category.

\section{Comparison between AG levels and impaired renal function (glomerular function)}

To determine the influence of impaired renal function on the plasma AG concentration, the correlation between the plasma AG and creatinine levels was ex- 


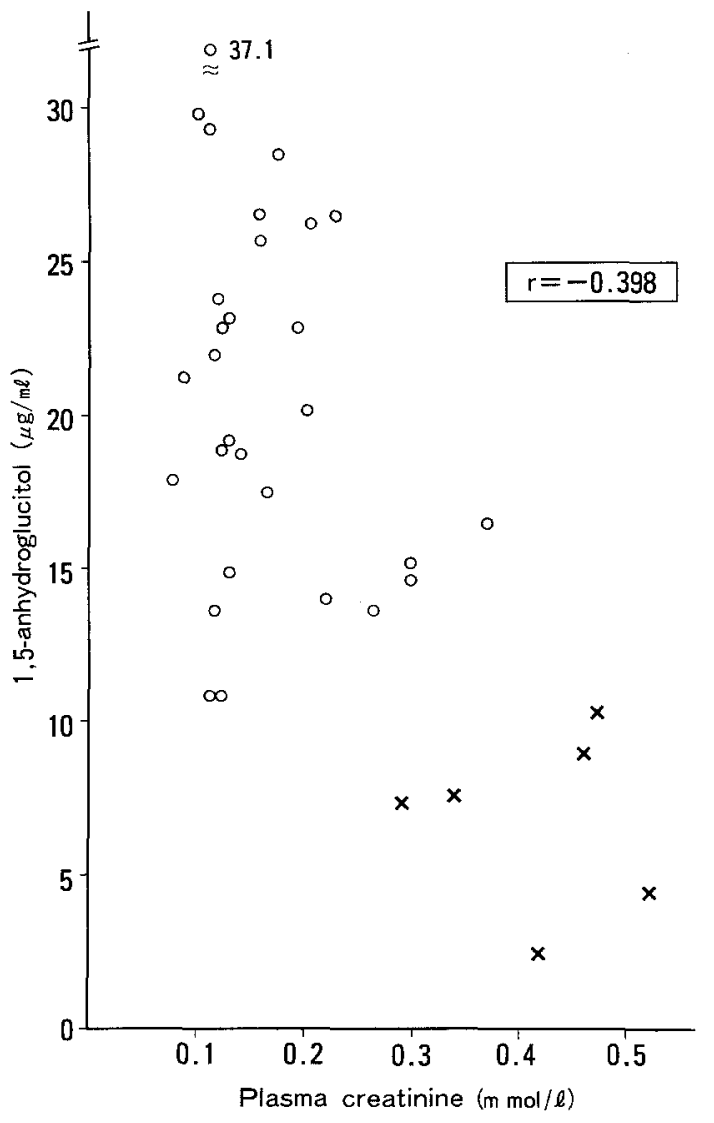

Fig. 1. The correlation between plasma creatinine and AG levels in 29 patients with impaired renal function (o) and 6 patients with severe renal failure accompanied by impaired glucose tolerance $(x)$. The patients with impaired renal function were the same subjects to those in Table 1

amined. In the patients with renal failure without diabetes mellitus, there was a relatively low correlation between the AG and creatinine concentrations in plasma $(r=-0.398, p<0.05)$ (Fig. 1). The plasma AG concentrations were higher than $10.8 \mu \mathrm{g} / \mathrm{ml}$ in all patients with renal failure without diabetes mellitus. In this study, five of six uraemic patients whose plasma creatinine level was more than $0.31 \mathrm{mmol} / 1$ accompanied with diabetes mellitus, and thus showed low plasma AG concentration.

\section{$A G$ concentration and obesity}

Table 2 shows the correlation of the plasma AG concentration with the degree of obesity in normal subjects. To exclude the influence of sex and age, all groups were statistically matched as to mean age and gender distribution. None of the differences in AG level among these obesity groups was significant. A similar result was obtained for the Type 2 diabetic patients (Table 3). With similar glycaemic control obtained by grouping patients according to $\mathrm{HbA}_{1}$ levels, obesity has little impact on the plasma AG concentration statistically. For this comparison, all groups were also matched as to mean age and gender distribution.
Table 2. Plasma 1,5-anhydroglucitol concentrations in obese or lean subjects

\begin{tabular}{lcl}
\hline $\begin{array}{l}\text { BMI } \\
\left(\mathrm{kg} / \mathrm{m}^{2}\right)\end{array}$ & Number & Plasma AG $(\mu \mathrm{g} / \mathrm{ml})$ \\
\hline $26.8 \pm 2.6$ & 7 & $20.1 \pm 8.5(\mathrm{NS})$ \\
$23.1 \pm 2.2$ & 23 & $22.5 \pm 8.8(\mathrm{NS})$ \\
$21.5 \pm 1.9$ & 51 & $22.0 \pm 8.4$ \\
$20.0 \pm 2.3$ & 30 & $24.8 \pm 8.2(\mathrm{NS})$ \\
$17.2 \pm 2.7$ & 11 & $23.2 \pm 7.4(\mathrm{NS})$ \\
\hline
\end{tabular}

Values are means \pm SD. BMI: body mass index

Table 3. Comparisons of the plasma AG concentration between obese and non-obese Type 2 (non-insulin-dependent) diabetic patients under similar glycaemic control

\begin{tabular}{llll}
\hline $\begin{array}{l}\mathrm{BMI} \\
\left(\mathrm{kg} / \mathrm{m}^{2}\right)\end{array}$ & \multicolumn{2}{l}{$\mathrm{HbA}_{1}$ (mean \%) } \\
\cline { 2 - 4 } & $\leqq 8.0$ & $8.1-10.0$ & $\geqq 10.1$ \\
\hline 25.0 & - & $\begin{array}{l}7.0 \pm 3.8(\mathrm{NS}) \\
(n=14)\end{array}$ & $\begin{array}{l}1.2 \pm 0.4(\mathrm{NS}) \\
(n=12)\end{array}$ \\
& & $\begin{array}{l}8.0 \pm 3.5(\mathrm{NS}) \\
(n=15)\end{array}$ & $\begin{array}{l}1.8 \pm 1.3(\mathrm{NS}) \\
(n=13)\end{array}$ \\
$\leqq 20.9$ & $15.9 \pm 3.5$ & $\begin{array}{l}6.4 \pm 3.0 \\
(n=25)\end{array}$ & $1.6 \pm 1.1$ \\
& $(n=19)$ & $(n=13)$ \\
\hline
\end{tabular}

The patients were selected as described under "Subjects". Plus-minus values are means $\pm S D$. Significance of difference compared with the group with similar $\mathrm{HbA}_{1}$ level. $\mathrm{BMI}$, body mass index.

\section{Dependence on age and sex}

The mean plasma AG concentrations showed a tendency to be higher in healthy males than in females in all age groups; however, statistically significant differences were not observed (data not shown). Also in neither the male nor the female group, was any significant influence of age observed. The highest AG level was found in males at ages $20-29$ years (mean \pm SD: $29.3 \pm 8.2 \mu \mathrm{g} / \mathrm{ml}$ ) and lowest in females at ages 10-19 years (mean \pm SD: $18.3 \pm 7.4 \mu \mathrm{g} / \mathrm{ml}$ ). In younger groups, it may be assumed that the plasma AG concentration has a tendency to be higher in males than females.

\section{Discussion}

The present results demonstrate that the concentration of plasma AG shows a specific reduction in diabetes mellitus. Although the decrease of plasma AG in diabetes mellitus has been confirmed $[7,8]$ and our previous report emphasized the sensitivity of this reduction to metabolic disarrangement, there is little information available on the specificity of the AG reduction in diabetes mellitus. We observed relatively low levels in malignancies, leukaemia, collagen diseases and insulinoma, whereas a relatively high level was seen in gout patients. These changes, however, were far less than that in diabetic patients, and would not interfere with 
the diagnosis of diabetes mellitus when the AG concentration was used as a marker of diabetes mellitus. The AG reduction in malignancies and leukaemia should be carefully assessed. Chronic malnutrition and/or a cachectic condition, or hyperalimentation may have some effect on the plasma AG concentration. Although the plasma AG concentration showed little diurnal change and was negligibly affected by either the diet [8] or short-term fasting (rat) (unpublished observation), there is little information available on the influence of prolonged starvation or hyperalimentation on the plasma AG concentration. Another possible cause of the low plasma AG concentration in malignant diseases is the concealed glucose-intolerant state. In this study, we carefully excluded patients with impaired glucose tolerance accompanying various diseases by checking the clinical history, randomly checked blood glucose concentrations, and 75-g GTT. However, in the malignancies and leukaemia groups, we could not apply this test, so non-diabetic subjects were judged only on the basis of randomly checked blood glucose concentrations. On the other hand, the $A G$ value was relatively high in gout patients. This difference may be partly due to the gender distribution of this group because the plasma AG concentration has a tendency to be higher in males than in females.

In a clinical study on uraemic patients, the plasma AG concentration was found to be almost within normal range and showed weak correlation with the plasma creatinine concentration. These observations suggested that the plasma AG concentration is little influenced by glomerular function. There have been some reports suggesting lower AG levels in CSF and plasma in uraemic patients than in healthy subjects [1, $9,11]$. This disagreement may be partly due to the inappropriate selection of uraemic patients. Niwa et al. reported [11] that $A G$ was significantly decreased in uraemic patients. However, four of their ten uraemic patients were undergoing haemodialysis treatment. Because the turnover rate of plasma $A G$ is known to be very low $[3,17]$, the rate of de novo AG synthesis is also expected to be very low. Therefore, the reduction in $A G$ in these cases seems likely to be simply due to the AG depletion by dialysis itself. Another possibility of the AG reduction in "uraemic patients" was due to the complication of impaired glucose tolerance. It is wellknown that patients with chronic renal failure show a very high incidence of glucose intolerance $[18,19]$. If patients with renal failure without glucose intolerance are strictly selected, the plasma AG concentration may not be low. This is actually supported by the data in Figure 1. The plasma AG concentration showed a tendency to decrease in parallel with development of glomerular dysfunction but the correlation coefficient for plasma $A G$ and creatinine was low, and the plasma AG distribution was within normal range in these patients. In the nephrotic syndrome, the mean plasma $A G$ concentration was $20.1 \pm 7.0 \mu \mathrm{g} / \mathrm{ml}(n=10)$, which was similar to that in healthy subjects. Based upon these observations, the phenomenon of remarkable AG reduction in uraemic patients is concluded to depend on additional factor(s).

Tables 2 and 3 indicate that the AG concentration is little influenced by the degree of obesity in both normal subjects and diabetic patients. AG seemed also to be little affected by fat metabolism, liver metabolism and hormones other than insulin [8]. Although the differences in plasma AG concentration between males and females were not significant, we got the impression that the AG level is generally higher in males than in females. Some unknown factor(s) may have some effect on the plasma AG level. However, this gender difference also may not interfere with the diagnosis of diabetes mellitus.

Although, until now, neither the physiological significance nor the pathway of metabolism of AG has been clarified, we have some evidence that the reduction of plasma AG in the diabetic condition is due to the accelerated urinary AG excretion which is coincidental with the excretion of glucose (unpublished data). The reduction of AG, one of the main polyols, may result in the occurrence of diabetic complications. Further studies are required to clarify these points.

In summary, this study demonstrated that the plasma AG level is not only sensitively but specifically reduced in diabetes mellitus. Since the plasma AG concentration is little influenced by various factors, such as lipid metabolism, liver dysfunction, renal dysfunction, various hormones other than insulin, body mass, sex or age, the metabolism and clearance of AG in the body may be affected by restricted factor(s) which are probably related to sugar metabolism and/or clearance.

Acknowledgements. This work was supported by Grants from Arima Memorial Foundation for Medical Research, and a Grant-in-Aid for Scientific Research (No.62570519) from the Ministry of Education, Science and Culture of Japan.

\section{References}

1. Pitkänen $E$ (1973) Occurrence of 1,5-anhydroglucitol in human cerebrospinal fluid. Clin Chem Acta 48: 159-166

2. Servo C, Pitkänen E (1975) Variation in polyol levels in cerebrospinal fluid and serum in diabetic patients. Diabetologia 11: $575-580$

3. Yamanouchi T, Akanuma H, Takaku F, Akanuma Y (1986) Marked depletion of plasma 1,5-anhydroglucitol, a major polyol, in streptozocin-induced diabetes in rats and the effect of insulin treatment. Diabetes 35: 204-209

4. Pitkänen E (1972) The serum polyol pattern and the urinary polyol excretion in diabetic and in uremic patients. Clin Chim Acta 38: $221-230$

5. Akanuma Y, Ogawa K, Yamanouchi T, Mashiko Y, Oka K, Kosaka K, Akanuma H (1981) Decreased plasma 1,5-anhydroglucitol in diabetic patients. Diabetes 30 [Suppl 1]: 124 A

6. Akanuma H, Ogawa K, Lee Y, Akanuma Y (1981) Reduced level of plasma 1,5-anhydroglucitol in diabetic patients. J Biochem 90: $157-162$ 
7. Yoshioka S, Saitoh S, Negishi C, Fujisawa T, Fujimori A, Takatani O, Imura M, Funabashi M (1983) Variations of 1-deoxyglucose (1,5-anhydroglucitol) content in plasma from patients with insulin-dependent diabetes mellitus. Clin Chem 29: 1396-1398

8. Yamanouchi $\mathrm{T}$, Akanuma $\mathrm{H}$, Asano $\mathrm{T}$, Konishi C, Akaoka I, Akanuma Y (1987) Reduction and recovery of plasma 1,5-anhydro-D-glucitol level in diabetes mellitus. Diabetes 36: 709-715

9. Servo C, Palo J, Pitkänen E (1977) Polyols in the cerebrospinal fluid and plasma of neurological, diabetic and uraemic patients. Acta Neurol Scandinav 56: 111-116

10. Pitkänen E (1982) Serum 1,5-anhydroglucitol in normal subjects and in patients with insulin-dependent diabetes mellitus. Scand J Clin Lab Invest 42: 445-448

11. Niwa T, Yamamoto N, Maeda K, Yamada K, Ohki T (1983) Gas chromatographic-mass spectrometric analysis of polyols in urine and serum of uremic patients. J Chromatogr 277:25-39

12. National Diabetes Data Group (1979) Classification and diagnosis of diabetes mellitus and other categories of glucose intolerance. Diabetes 28: 1039-1057

13. WHO Expert Committee on Diabetes Mellitus (1980) Second report, Technical Report Series 646 Geneva

14. Hsieh S, Akanuma Y (1985) Instability of fasting blood glucose values in noninsulin-dependent diabetic patients with long-term insulin treatment. Metabolism 34: 371-376
15. Ohkubo A, Kamei S, Yamanaka M, Arai F, Kitajima M, Kondo A (1981) Plasma glucose concentrations of whole blood, as determined with a multilayer-film analytical element. Clin Chem 27: $1287-1290$

16. Poulsen S, Billesbolle P, Kolendorf K, Thorsteinsson B (1985) The C-peptide response to glucagon injection in IDDM and NIDDM patients. Horm Metabol Res 17: 39-40

17. Pitkänen E, Pitkänen $O$ (1984) The elimination of 1,5-anhydroglucitol administered to rats. Experientia 40: 463-465

18. Westervelt FB, Schreiner GE (1962) The carbohydrate intolerance of uremic patients. Ann Intern Med 57: 266-276

19. Spitz IM, Rubenstein AH, Bersohn I, Abrahams C, Lowy C (1970) Carbohydrate metabolism in renal disease. Quart J Med NS 39: 201-226

Received: 25 May 1987

and in revised form: 2 November 1987

Dr. Toshikazu Yamanouchi

The Second Department of Internal Medicine

University of Teikyo

Itabashiku Tokyo 173

Japan 